\title{
The Efficacy of Visual Activity Schedule Intervention in Reducing Problem Behaviors in Children With Attention-Deficit/Hyperactivity Disorder Between the Age of 5 and 12 Years: A Systematic Review
}

\author{
Naveena Thomas and Sudhin Karuppali \\ Department of Audiology and Speech Language Pathology, Kasturba Medical College, Mangalore, Manipal Academy of Higher Education, \\ Manipal, Karnataka, India
}

\begin{abstract}
Objectives: Children with attention-deficit/hyperactivity disorder (ADHD) tend to be noisy and violate rules with their disruptive behaviors, resulting in greater difficulties with off-task behaviors and being at risk for social refusal. The visual activity schedule (VAS) intervention program is a frequently used method to teach multiple skills involving on-task, use of schedules, transition behaviors, social initiation, independent play skills, classroom skills, and academic skills. The current systematic review aimed to examine the efficacy of using VAS intervention in reducing problem behaviors in children with ADHD between 5 and 12 years of age.

Methods: Systematic searches were conducted using two electronic databases (PubMed and Scopus) to identify relevant studies published in English between 2010 and 2020. Four studies met the inclusion criteria: two studies examined the effect of schedule-based tasks and the use of an iPad on classroom skills, while the other two examined randomized clinical trials (RCTs) of psychosocial treatment for ADHD inattentive type and a cross-sectional study examined the impact of the group size on task behavior and work productivity in children with ADHD.

Results: The findings indicate that the interventions used in all four studies could lead to increased satisfaction among participants and parents, as well as a reduction in problem behavior. In terms of the research indicators, the RCT had low quality, while the others were of high quality.

Conclusion: A larger number of studies and the ADHD clinical population would help to increase the generalizability of future reviews of treatments in this context.
\end{abstract}

Keywords: Attention deficit hyperactivity disorder; Schedule; Social; Problem; Behaviors.

Received: July 21, 2021 / Revision: September 22, 2021 / Accepted: September 30, 2021

Address for correspondence: Sudhin Karuppali, Department of Audiology and Speech Language Pathology, Kasturba Medical College, Mangalore, Manipal Academy of Higher Education, Manipal, Karnataka, India

Tel: +91-9844807634, Fax: +91-8242428183, E-mail: sudhin.karuppali@manipal.edu

\section{INTRODUCTION}

Attention-deficit/hyperactivity disorder (ADHD) is a condition that occurs in children during the developmental period with symptoms being manifested at home and school with characteristics such as impulsivity, inattention, and hyperactivity [1]. ADHD syndrome has been categorized into three types, namely: the combination type, the predominantly inattentive type, and the predominantly hyperactive/impulsive type. The severity of the symptoms ranges from mild, moderate, and severe (American Psychiatric Association, 2013) [2]. The Diagnostic and Statistical Manual of Mental

This is an Open Access article distributed under the terms of the Creative Commons Attribution Non-Commercial License (https://creativecommons.org/licenses/by-nc/4.0) which permits unrestricted non-commercial use, distribution, and reproduction in any medium, provided the original work is properly cited.
Disorders, 5th edition (DSM-5) mentions that people with ADHD have a history of inattention and/or hyperactivityimpulsivity, which makes it difficult for them to work and develop skills [2]. According to the DSM-5, individuals with ADHD under the age of 16 should exhibit six or more symptoms of inattention and hyperactivity-impulsivity, while adolescents and adults aged 17 and above should exhibit five or more of the symptoms, with the symptoms having been present for at least six months, which becomes inadequate for their developmental stage. Although some of the abovementioned behaviors are sometimes considered as characteristic features of typically developing children as well, when exhibited inappropriately in varied settings, it is reflected as a psychiatric disorder [1].

Children with ADHD tend to be noisy and violate rules with 
their disruptive behaviors, resulting in greater difficulties with off-task behaviors, thereby bringing them at risk for social refusal [3]. They tend to exhibit signs of hyperactivity and inattention, coexisting with a reduced level of communication skills [4]. Pronounced deficits in phonological and syntactic structures have been noted in children with ADHD [5], which is crucial for literacy development [6]. Apart from these linguistic components, studies have reported language problems in ADHD characterized by pragmatic deficits [7]. These pragmatic impairments associated with ADHD may include children having: 1) reduced verbal output and further disfluencies when faced with activities that include preparation and coordination of verbal responses, such as storytelling or giving directions; 2) timing issues when it comes to starting a discussion, taking turns and keeping or changing topics during a conversation; and 3) excessive verbal output during spontaneous interactions, task changes, and play conditions [8].

Different interventional approaches have been suggested for the treatment of individuals with ADHD, which may include behavioral, pharmacological, psychotherapeutic, physical exercise, and education-based approaches [9]. Pelham et al. [10] emphasized the role of behavioral interventions for ADHD, which included parents and teachers administered, combined, and direct intervention programs with the affected individuals. Parents become primary educators in their children's lives, with the power to mold their attitudes. As a result, it is important to provide parents with the support they need by addressing their child's problem habits and offering solutions for modifying or enhancing the behaviors that interfere with their daily activities. Rather than focusing on the symptoms of ADHD, a behavioral approach focuses on functional impairments [11]. Some of the key organizational skills required for children with ADHD have been noted to be in the area of strategy and skill preparation [12].

School-home note intervention is a frequently used method to enhance classroom behavior and task-relevant behavior in middle-aged and elementary school children [13]. Using methods such as these have resulted in a significant enhancement in classroom behaviors such as paying attention, performing homework, talking with the teacher's permission, maintaining seating, as well as executing quality and quantity classwork [13]. As children with ADHD face challenges in academic settings, it becomes difficult for them to perform well in classroom settings, with frequent display of symptoms of inattention, disruptive behaviors, poor sitting behaviors and an inability to control their impulses. The visual activity schedule (VAS) intervention program is a frequently used method to teach multiple skills involving on-task, use of schedules, transition behaviors, social initiation, independent play skills, classroom skills, and academic skills [14]. An activity schedule can include pictures, drawings, photographs, or a set of images that cue a person to engage in a sequence of doing things that helps one to complete their activity or assignment without the need for assistance. A child's academic and social skills depend on their ability to remain on tasks with minimal distractions. Such skills allow children to gain the required input, complete tasks or assignments, and be active during discussions that are held in a classroom [15]. VASs have been used to reduce the latency to initiate a new activity [16], reduce temper tantrums during transitions [17], and to enhance and maintain multiple social skills [14]. The implementation of VAS intervention programs such as providing smallgroup directions have been used in general classrooms and/ or educational settings [18], aiding in the enhancement of academic skills and the degree of interaction between peers, thereby reducing disruptive behaviors in classrooms [19]. This interventional approach is commonly used among children with autism spectrum disorder (ASD) [20] and intellectual disabilities [21]. Children diagnosed with ADHD exhibit difficulties in these skills, leading to disruptive behaviors in the classroom. The efficacy of using the VAS in children with ADHD has been studied minimally compared to that in children with ASD. Consequently, the current systematic review aimed to evaluate the efficacy of using the VAS in children with ADHD between the ages of 5 and 12 years.

\section{METHOD}

\section{Protocol and registration}

The review protocol for the current study was registered in the International Prospective Register of Systematic Review (PROSPERO). The registered protocol can be found at: https://www.crd.york.ac.uk/prospero/display_record. php?RecordID=212844.

\section{Electronic search strategy}

The databases used for the present systematic review included the PubMed and Scopus databases. A search strategy was used for each of the databases with two keywords pertaining to the population ("attention deficit hyperactivity disorder," "ADHD"); seven keywords in relation to the intervention ("VAS," "visual activity schedule," "picture prompts," "visual schedule," "visual cues," "work system," "visual reminders"); and five keywords pertaining to the outcome ("on task behavior," "on schedule behavior," "independent skills," "social skills," "problem behaviors") [14,22-26], with the Boolean operators such as "AND," "OR," and "NOT."

The initial search was performed on October 16, 2020, and the search terms were determined based on two criteria. First, 
the outcomes or skills that improved with the use of VAS were identified from eight research articles [14,22-28] with outcomes being considered as the key words. Second, most of the possible alternative words were used as keywords in the search to avoid missing any relevant articles. The search was restricted to peer-reviewed English language studies published between 2010 and 2020 . We also manually searched for the following journals: 1) Journal of Emotional and Behavioral Disorders; 2) Journal of Behavioral Neurology; 3) Journal of Psychology \& Psychotherapy; 4) Journal of Health \& Medical Research; and 5) Journal of Special Education and Technology to identify any potential articles that could have been missed.

\section{Study selection}

A three-phase selection process was considered for the final inclusion of the studies. It was initiated by compiling the studies obtained from the search of the two databases using the Mendeley desktop reference management system. In the second phase, the duplicates were removed, and using the COVIDENCE manager (https://www.covidence.org/) the two reviewers independently screened the titles and abstracts according to the inclusion and exclusion criteria set a priori. The inclusion criteria were: 1) children diagnosed with ADHD in the age range of 5 and 12 years; 2) English language peerreviewed observational studies or randomized controlled trials (RCTs) published between 2010 and 2020; 3) either single case research studies or group design studies; 4) studies with at least one participant diagnosed with ADHD; and 5) studies from PubMed and Scopus databases. The exclusion criteria were as follows: 1) children diagnosed with other disabilities such as ASD and intellectual disabilities; 2) children with neurodevelopmental disorders; 3) thesis/dissertations from institutional repositories; 4) narrative review articles, case reports, or editorials; and 5) studies with adolescents with ADHD (over 12 years of age).

The full length review was performed only after we identified all relevant articles, including the manual search. In cases of uncertainty over whether an abstract met the inclusion criterion, the authors obtained the full-text article and independently evaluated each paper to incorporate it. In the event of a disagreement, the researchers arrived at a consensus by reevaluating the inclusion criterion and recorded the reasons for excluding these studies. Finally, we recorded our decisionmaking process using a Preferred Reporting Items for Systematic Review and Meta-Analyses (PRISMA) chart [29].

\section{Data extraction and management}

The two reviewers independently extracted the data from the full-length articles that met our criteria using a specifi- cally devised data extraction form. A third reviewer checked both of the reviewers' data extractions. The following data were extracted for the current systematic review: 1) research design; 2) participant gender and age; 3) settings; 4) targeted skills; 5) dependent variables/measures; 6) schedule type and mode of presentation; 7) materials used; 8) strategies used; and 9) results/outcomes [14].

\section{Quality assessment}

The quality of the two single case studies $[25,26]$ was assessed using relevant quality indicators [28]. The quality assessment scale, a yes/no rated system, was used to evaluate if the described criteria were met. A total of 20 indicators were used to assess the quality of the reviewed studies. Two independent reviews assessed the quality of each study using the quality indicators [28], and responses were verified by a third reviewer. One of the studies by Hart et al. [27], followed a crosssectional case design, and the quality (selection, comparability, and outcome) of the study was assessed using the Newcastle Ottawa scale [30]. The quality of the fourth study by Pfiffner et al. [31] followed a randomized clinical trial which was assessed using the validated revised Cochrane risk-ofbias tool for randomized trials (RoB 2) [32].

\section{RESULTS}

\section{Study selection}

Initially, 964 articles were identified across the two databases. Among them, 444 were found to be duplicates and were removed based on the title and abstract. The existing 525 articles were screened, and 489 were found to be irrelevant based on the prior set of the inclusion and exclusion criteria set by the researchers. A total of 57 articles met the eligibility criteria, of which 53 articles were excluded after full length review. Finally, four articles were found to be relevant to the study and met the inclusion criteria. The PRISMA chart of the systematic review is shown in Fig. 1.

\section{Study characteristics}

The study characteristics of the four included articles have been shown in Table 1.

\section{Participants}

Among the four studies, 237 children were diagnosed with ADHD, with $38.81 \%$ being female and $61.18 \%$ being male. All participants in the four studies were between 5 and 12 years of age. Two studies reported recruiting 58\% [31] and 76\% [27] of male participants. The 199 participants included in one of the studies [31] included children between 7 and 11 years of age, diagnosed with ADHD-Inattentive Type (AD- 


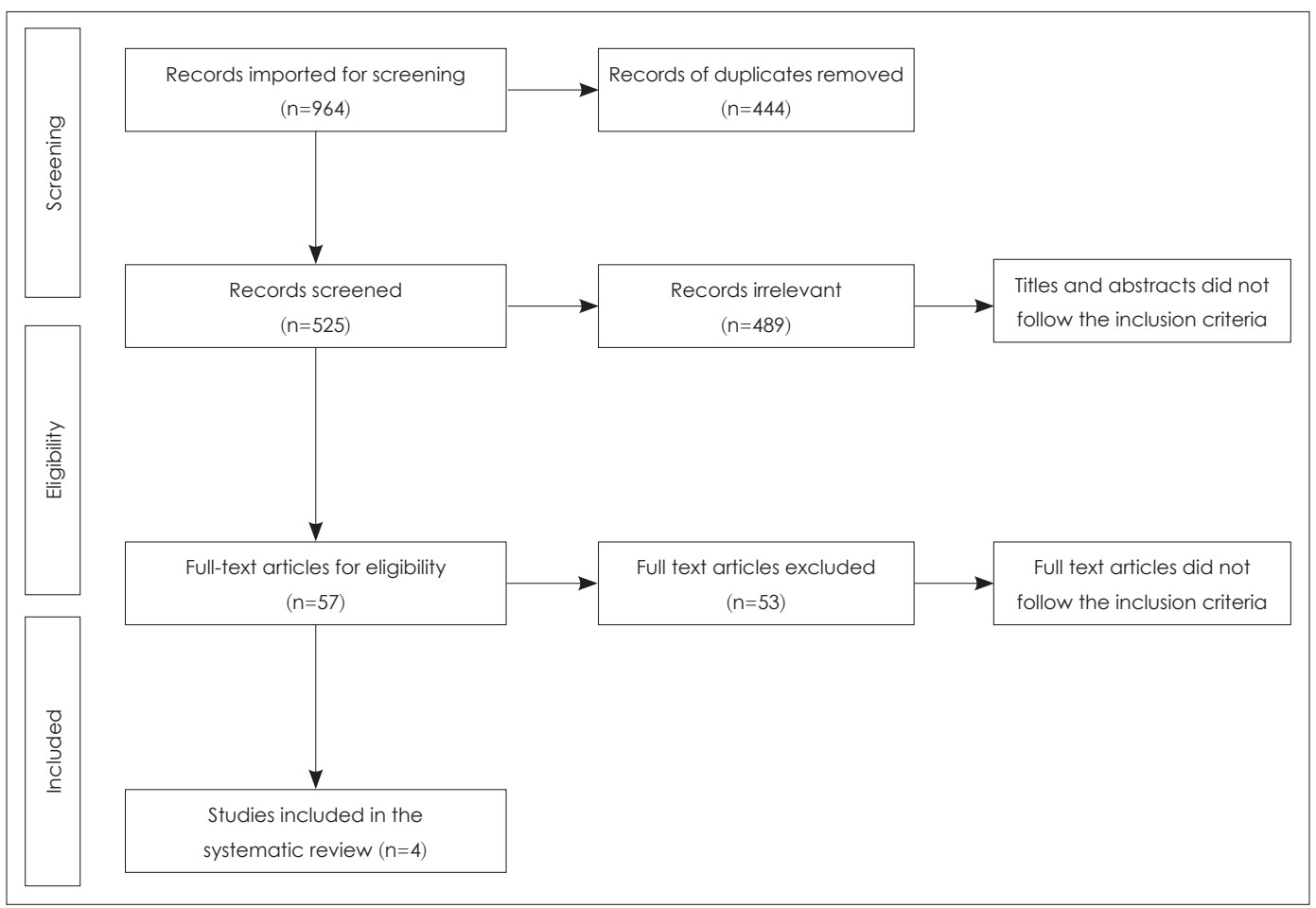

Fig. 1. The Preferred Reporting Items for Systematic Review flowchart for the current study.

HD-I); and in another study [27], the 33 participants included children between 7 and 12 years of age who were diagnosed with ADHD. Among the two single case studies, one of them [26] reported two male participants of 7 and 9 years of age, respectively, while the other [25] reported three male participants (a child with moderate intellectual disability, a child with ADHD, and a child with global delay) with the ADHD participant aged 5.8 years.

All four selected studies had at least one child with ADHD who participated in the study. In one of the single case studies [26], the risk of ADHD in the two participants was rated using Conner's Teacher Rating Scales-Revised, Short and Conner's Parent Rating Scales-Revised, Short [33]. In another study [25], there was no mention of the scale/tool used to diagnose the participant as ADHD. Since the use of a standard diagnostic system was not part of the study selection criteria for the current systematic review, this study [25] was therefore considered for the review, primarily because it met all other criteria pertaining to the use of VAS as an intervention for children with ADHD.

In a RCT study [31], the participants were diagnosed with ADHD-I using the Kiddie Schedule for Affective Disorders and Schizophrenia for School-Age Children and Wechsler Intelligence Scale for Children and for ADHD-I, all of the patients met the full DSM-IV requirements. In a study by Hart et al. [27], the researchers used parent and teacher rating scales, such as the Disruptive Behavior Rating Scale [34] and IOWA
Conners Rating Scale [35] to determine ADHD symptoms.

\section{Settings}

All four studies conducted the intervention program in different settings and/or environments [25-27,31]. Two single case studies $[25,26]$ conducted the treatment in the participant's classroom. A RCT study [31] conducted an intervention at the University of California San Francisco and the University of California, Berkeley. The intervention included group meetings with parents and children in clinics, the school premises, and over the phone, while the teacher consultations were conducted at the school and occasionally over the phone. Hart et al. [27] conducted a study relating to a summer treatment program in an academic learning center. $\mathrm{Ci}$ relli et al. [26] mentioned the layout of the classroom, which included 20 desks for students, one desk for teachers and a horseshoe-shaped table. However, none of the other studies $[25,27,31]$ reported the same.

\section{Quality assessment}

The quality of the two single case studies $[25,26]$ was assessed using single-case study quality indicators [28]. The quality of the study was determined by taking the ratio of the total number of agreements by the total number of indicators, and then multiplying by 100 . One of the single case studies [26] received a quality appraisal of $95 \%$, while the study by Stephenson [25] met a 90\% quality standard, as shown in 
Table 1. Data extraction sheet of four articles included in the systematic review

\begin{tabular}{|c|c|c|c|c|}
\hline Characteristics & Cirelli et al. [26] & Pfiffner et al. [31] & Stephenson [25] & Hart et al. [27] \\
\hline $\begin{array}{l}\text { Research } \\
\text { design }\end{array}$ & $\begin{array}{l}\text { Nonconcurrent multiple } \\
\text { baseline design }\end{array}$ & $\begin{array}{l}\text { Randomized } \\
\text { controlled trial }\end{array}$ & Multiple baseline & Not reported \\
\hline Participants & $\begin{array}{l}\text { Participants: } 2 \text { males } \\
\text { of } 7 \text { and } 9 \text { years; } \\
\text { Diagnosis: attention- } \\
\text { deficit/hyperactivity } \\
\text { disorder (ADHD) }\end{array}$ & $\begin{array}{l}\text { Participants: } 199 \text { ( } 58 \% \text { of } \\
\text { males) of } 7-11 \text { years; } \\
\text { Diagnosis: ADHD-Inattentive } \\
\text { Type }\end{array}$ & $\begin{array}{l}\text { Participants: } 3 \text { males } \\
\text { 1) } 6.3 \text { years; diagnosis: } \\
\text { autism and } \\
\text { developmental disability } \\
\text { with moderate intellectual } \\
\text { disability } \\
\text { 2) } 5.8 \text { years; diagnosis: } \\
\text { moderate intellectual } \\
\text { disability, ADHD, and } \\
\text { global delay } \\
\text { 3) } 5.5 \text { years; diagnosis: } \\
\text { autism spectrum disorder }\end{array}$ & $\begin{array}{l}\text { Participants: } 33 \text { ( } 76 \% \text { of } \\
\text { males) of } 7-12 \text { years; } \\
\text { Diagnosis: ADHD }\end{array}$ \\
\hline Setting & $\begin{array}{l}\text { Each participant's } \\
\text { classroom }\end{array}$ & $\begin{array}{l}\text { University of California } \\
\text { San Francisco; University } \\
\text { of California, Berkeley }\end{array}$ & Classroom & $\begin{array}{l}\text { Academic learning } \\
\text { center }\end{array}$ \\
\hline $\begin{array}{l}\text { Targeted skills/ } \\
\text { depended } \\
\text { measures }\end{array}$ & $\begin{array}{l}\text { On-task behavior and } \\
\text { on-schedule behavior }\end{array}$ & $\begin{array}{l}\text { Skills: organizational, social, } \\
\text { independent, academic, } \\
\text { self-care, daily living }\end{array}$ & $\begin{array}{l}\text { Reading, writing, numbers, } \\
\text { special activity }\end{array}$ & $\begin{array}{l}\text { On task behavior and } \\
\text { work productivity } \\
\text { (academic skills, reading } \\
\text { skills, independent skills) }\end{array}$ \\
\hline $\begin{array}{l}\text { Type of } \\
\text { intervention }\end{array}$ & $\begin{array}{l}\text { Activity schedule and } \\
\text { through worksheet, } \\
\text { schedule book, } \\
\text { written instructions } \\
\text { on paper strips, } \\
\text { visual organizational } \\
\text { charts, sticker charts }\end{array}$ & $\begin{array}{l}\text { Child Life and Attention } \\
\text { Skills Treatment: } \\
\text { - Ten 90-minute parent } \\
\text { group meetings } \\
\text { - Six 30-minute child group } \\
\text { meetings } \\
\text { - Teacher consultation } \\
\text { Parent-focused treatment: } \\
\text { - Parent training group } \\
\text { - Individual family meetings } \\
\text { - Monthly treatment booster } \\
\text { sessions for families } \\
\text { Treatment as usual: } \\
\text { - Parenting workshop } \\
\text { - Special education services } \\
\text { - Seating modified homework } \\
\text { - Behavioral chart } \\
\text { - Extra time on tests }\end{array}$ & $\begin{array}{l}\text { Schedule apps through } \\
\text { iPads and pictures } \\
\text { displayed }\end{array}$ & $\begin{array}{l}\text { Summer treatment } \\
\text { program: small-group } \\
\text { condition } \\
\text { - Reading comprehension } \\
\text { activity in the } \\
\text { instructional period } \\
\text { - Reading comprehension } \\
\text { testing during testing } \\
\text { period whole-group } \\
\text { condition } \\
\text { - Reading comprehension } \\
\text { activity during } \\
\text { instructional period } \\
\text { - Reading comprehension } \\
\text { test during testing } \\
\text { period } \\
\text { Independent seatwork } \\
\text { - Reading comprehension } \\
\text { activity during } \\
\text { instructional period } \\
\text { - Reading comprehension } \\
\text { test during testing period }\end{array}$ \\
\hline Strategies used & $\begin{array}{l}\text { Simplified directions, } \\
\text { repetition of directions, } \\
\text { incentive coupons, } \\
\text { extended time, } \\
\text { rephrasing questions }\end{array}$ & $\begin{array}{l}\text { Problem-solving steps, } \\
\text { self-cues, reminder list, } \\
\text { role plays, scaffolding, } \\
\text { routinization, using rewards, } \\
\text { positive consequence such } \\
\text { as praises, cue based } \\
\text { reminders, organizational } \\
\text { strategies, group based } \\
\text { rewards, play activities }\end{array}$ & $\begin{array}{l}\text { Verbal and visual } \\
\text { prompts }\end{array}$ & Not reported \\
\hline $\begin{array}{l}\text { Results/ } \\
\text { outcomes }\end{array}$ & $\begin{array}{l}\text { Increased on task and } \\
\text { on schedule behavior } \\
\text { in the absence of } \\
\text { teacher prompts }\end{array}$ & $\begin{array}{l}\text { Reduction of inattention } \\
\text { symptoms, increases in } \\
\text { social skills, and overall } \\
\text { improvement, increased } \\
\text { organizational skills }\end{array}$ & $\begin{array}{l}\text { Completed one step in the } \\
\text { intervention session and } \\
\text { improved rapidly in terms } \\
\text { of schedule app usage } \\
\text { through writing, reading } \\
\text { and number based } \\
\text { activities }\end{array}$ & $\begin{array}{l}\text { Increased on-task } \\
\text { behavior during } \\
\text { small-group instruction, } \\
\text { increased work } \\
\text { productivity during small } \\
\text { group condition }\end{array}$ \\
\hline
\end{tabular}


Table 2. Quality checklist for single case studies (quality indicators of single case designs)

\begin{tabular}{|c|c|c|}
\hline Indicators & Cirelli et al. [26] & Stephenson [25] \\
\hline \multicolumn{3}{|l|}{ Participant } \\
\hline Described sufficiently & Y & Y \\
\hline Selection described sufficiently & $\mathrm{N}$ & $\mathrm{N}$ \\
\hline Setting described sufficiently & Y & Y \\
\hline \multicolumn{3}{|l|}{ Dependent variable (DV) } \\
\hline Described with replicable precision & Y & Y \\
\hline Quantifiable & Y & Y \\
\hline Measurement described to replicable precision & Y & Y \\
\hline Measurement occurred repeatedly & Y & Y \\
\hline Interobserver agreement data reported & Y & Y \\
\hline \multicolumn{3}{|l|}{ Independent variable (IV) } \\
\hline Described with replicable precision & Y & Y \\
\hline Systematically manipulated & Y & Y \\
\hline Procedural fidelity described & Y & Y \\
\hline \multicolumn{3}{|l|}{ Baseline } \\
\hline Phase provided evidence of pattern, prior to intervention & Y & Y \\
\hline Described with replicable precision & Y & Y \\
\hline \multicolumn{3}{|l|}{ Validity } \\
\hline Three demonstrations of experimental effect & Y & Y \\
\hline Design controlled threats to internal validity & Y & Y \\
\hline Effects replicated, indicate external validity & Y & Y \\
\hline DV socially important & Y & Y \\
\hline Magnitude of change in DV due to intervention socially important & Y & Y \\
\hline IV is cost effective/practical & Y & $\mathrm{N}$ \\
\hline IV is implemented over time, typical contexts/typical agents & Y & Y \\
\hline Indicators met/total indicators & 19 & 18 \\
\hline Total percentage & $19 / 20 \times 100=95 \%$ & $18 / 20 \times 100=90 \%$ \\
\hline
\end{tabular}

Table 2. The ratings obtained for the cross-sectional study [27] for each section (selection, comparability, and outcome) were 4,1 , and 3 , respectively, indicating good quality, as shown in Table 3. For the RCT study [31], the quality was determined by taking the ratio of the total number of "yes" (5) and the total indicators (19), then multiplying by 100 . The result revealed a score of $26.3 \%$, suggesting the study was of low quality, as shown in Table 4.

The treatment procedure of the two single case studies $[25,26]$ is illustrated in Table 5 .

The treatment procedure of the RCT [31] and cross-sectional study design [27] are illustrated in Table 6.

\section{DISCUSSION}

The current systematic review included four peer-reviewed articles (two single case studies, one cross-sectional study, and one RCT study). All four studies attempted to focus on ADHD participants, with the single case studies $[25,26]$ having a smaller number of participants which enabled them to provide more attention to the chosen participants. This was possible in the RCT [31] and the cross-sectional study [27] because of the large number of children who participated. One of the parameters set for the study selection criteria was to have at least one participant diagnosed with ADHD, which was met by all four studies that were finally included in this systematic review. However, in the study by Stephenson [25], although one of the participants was diagnosed with ADHD, there was no mention of the diagnostic system used for the same. However, as the use of a standard diagnostic system was not part of the study selection criteria for the current systematic review, this study [25] was considered for the review, primarily because it met all other criteria pertaining to the use of VAS as an intervention for children with ADHD. The recruitment of participants [36] and the study settings [37] largely contributed to the efficacy of the study. The recruitment process was well-documented in the RCT and the crosssectional study, but not in the two single case studies. Similarly, the study settings in the two single case studies were mentioned clearly, since it was conducted in a school and 
Table 3. Quality checklist for cross sectional study (Newcastle Ottawa Scale [30])

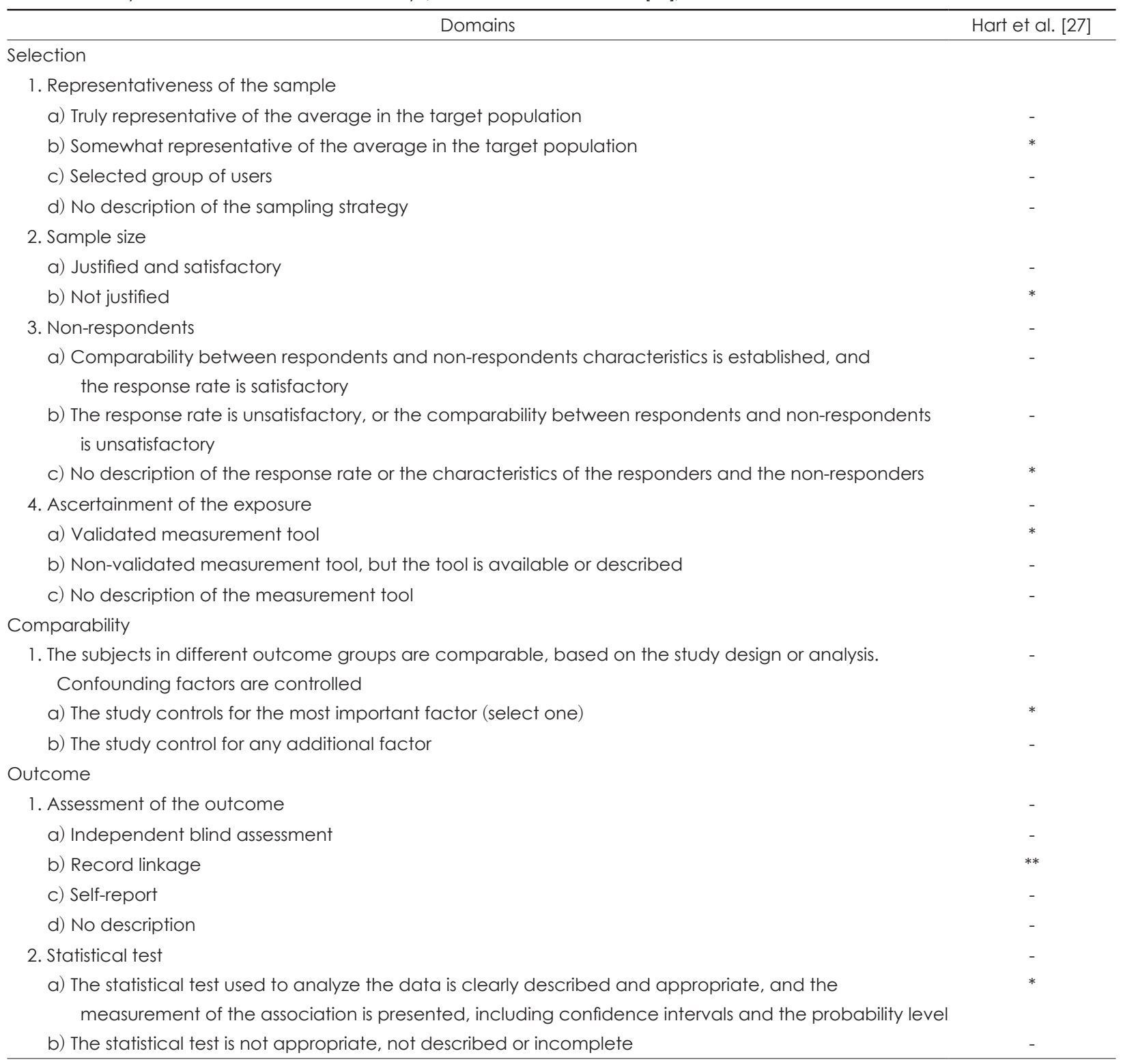

classroom setting; however, there was no such clarification in the RCT and cross-sectional study. As the single case studies $[25,26]$ were conducted in the classroom, the examiners were able to concentrate on classroom and academic skills that the participants could easily generalize [38]. In a crosssectional study [27], academic testing was used as part of the recruiting procedures to assess the participants' eligibility for the study, which was not addressed in other studies, however, it is generally considered as an ideal method for recruiting participants for research studies [39].

Prior to the treatment sessions, the screening protocol was discussed in terms of interviews with both parents and teachers [31]; however, the diagnostic procedures were mentioned in the cross-sectional study but not in the two single case studies. In all four studies [25-27,31], the chosen participants were assessed on a variety of skills. Two of the single case studies centered on classroom skills, while the RCT focused on everyday living skills. Hart et al. [27] targeted task behavior, which was observed over two periods. Despite the fact that the four studies focused on a wide range of skills, all of the targeted skills were significant in improving abilities and decreasing problem behaviors. In terms of the practices and materials used, each of the four studies took a different approach towards intervention. The materials used in the three studies [25-27] differed based on the type of intervention program. Pfiffner et al. [31] did not report the materials used in the session, even though the intervention had been mentioned. A variety of strategies, including verbal, gestural, as 
Table 4. Quality checklist for randomized controlled trial [31] (Revised Cochrane risk-of-bias tool for randomized trials [RoB 2] [32])

\begin{tabular}{|c|c|c|}
\hline SI. no & Signaling questions & Response options \\
\hline \multicolumn{3}{|c|}{ Risk of bias arising from the randomization process } \\
\hline 1. & Was the allocation sequence random? & Y/PY/PN/N/NI \\
\hline 2. & $\begin{array}{l}\text { Was the allocation sequence concealed until participants were enrolled and assigned } \\
\text { to interventions? }\end{array}$ & Y/PY/PN/N/NI \\
\hline 3. & $\begin{array}{l}\text { Did baseline differences between intervention groups suggest a problem with the randomization } \\
\text { process? }\end{array}$ & Y/PY/PN/N/NI \\
\hline
\end{tabular}

Risk of bias due to deviations from the intended intervention/effect of assignment/adhering to intervention

4. Were participants aware of their assigned intervention during the trial?

Y/PY/PN/N/NI

5. Were carers and people delivering the interventions aware of participant's assigned intervention during the trial?

6. Were important non-protocol interventions balanced across intervention groups

Y/PY/PN/N/NI

7. Were there deviations from the intended intervention that arose because of the trial context

NA/Y/PY/PN/N/NI

8. Was an appropriate analysis used to estimate the effect of assignment/adhering to intervention?

NA/Y/PY/PN/NI

Y/PY/PN/N/NI

9. Was there potential for a substantial impact (on the result) of the failure to analyze participants

NA/Y/PY/PN/N/NI in the group to which they were randomized?

Risk of bias due to missing outcome data

10. Were data for this outcome available for all, or nearly all, participants randomized?

Y/PY/PN/N/NI

11. Is there evidence that the result was not biased by missing outcome data? NA/Y/PY/PN/N/NI

Risk of bias in measurement of the outcome

12. Was the method of measuring the outcome inappropriate?

Y/PY/PN/N/NI

13. Could measurement or ascertainment of the outcome have differed between intervention groups?

Y/PY/PN/N/NI

14. Were outcome assessors aware of the intervention received by study participants?

15. Could assessment of the outcome have been influenced by knowledge of intervention received? NA/Y/PY/PN/N/NI $\mathrm{NA} / \mathrm{Y} / \mathrm{PY} / \mathrm{PN} / \mathrm{N} / \mathrm{NI}$

16. Is it likely that the assessment of the outcome was influenced by knowledge of intervention $\mathrm{NA} / \mathrm{Y} / \mathrm{PY} / \mathrm{PN} / \mathrm{N} / \mathrm{NI}$ received?

Risk of bias in selection of the reported result

17. Were the data that produced this result analyzed in accordance with a pre-specified analysis plan that was finalized before unblinded outcome data were available for analysis?

18. Is the numerical result being assessed likely to have been selected, on the basis of the results, from multiple eligible outcome measurements (e.g., scales, definitions, time points) within the outcome domain?

19. Is the numerical result being assessed likely to have been selected, on the basis of the results, from multiple eligible analyses of the data?

Y, yes; PY, probably yes; PN, probably no; N, no; NI, no information

well as gestural and verbal prompts, were used in the schedule-based intervention program in one of the single case studies, while both verbal and physical prompts were used in another study [25]. Physical prompts were discovered to have a significant impact on eliciting the responses, which was not mentioned in RCTs [31] or cross-sectional studies [27]. During any treatment program, the duration of the session played a significant role. The duration mentioned in all four studies was different, and depended on how sufficiently they had used the strategies, tasks, and materials for the participants. In the study conducted by Cirelli et al. [26], the students were required to complete worksheets and place them in a work folder, which took a longer time (25-30 minutes) than the participants in the Stephenson [25] study who were expect- ed to complete basic activities (counting tasks, letter identification, and numbering) using an iPad, which took a shorter time (5-10 minutes). The Child Life and Attention Skills Treatment (CLAS) program used in the study by Pfiffner et al. [31] lasted longer as it focused on the training of parents, teachers, and children with ADHD. In addition, the intervention program used here included parent-focused treatment and treatment as usual. Therefore, the amount of time needed to achieve the desired level of satisfaction was longer. The details of the program duration are listed in Table 6. In the study by Hart et al. [27], the authors provided an intervention program (10 minutes each) under three different conditions (whole group, small group, and independent seatwork), which also resulted in a longer duration of VAS inter- 


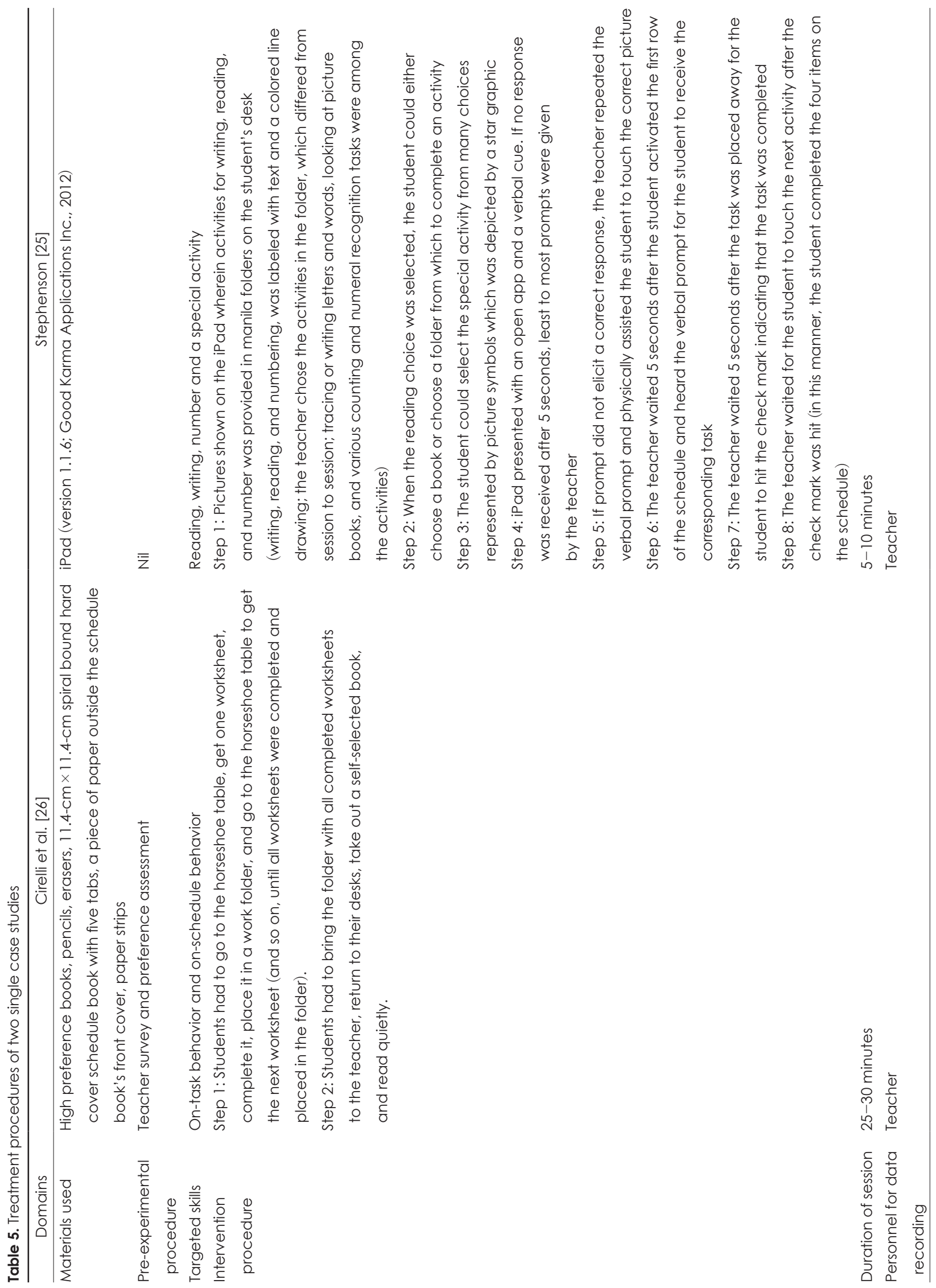




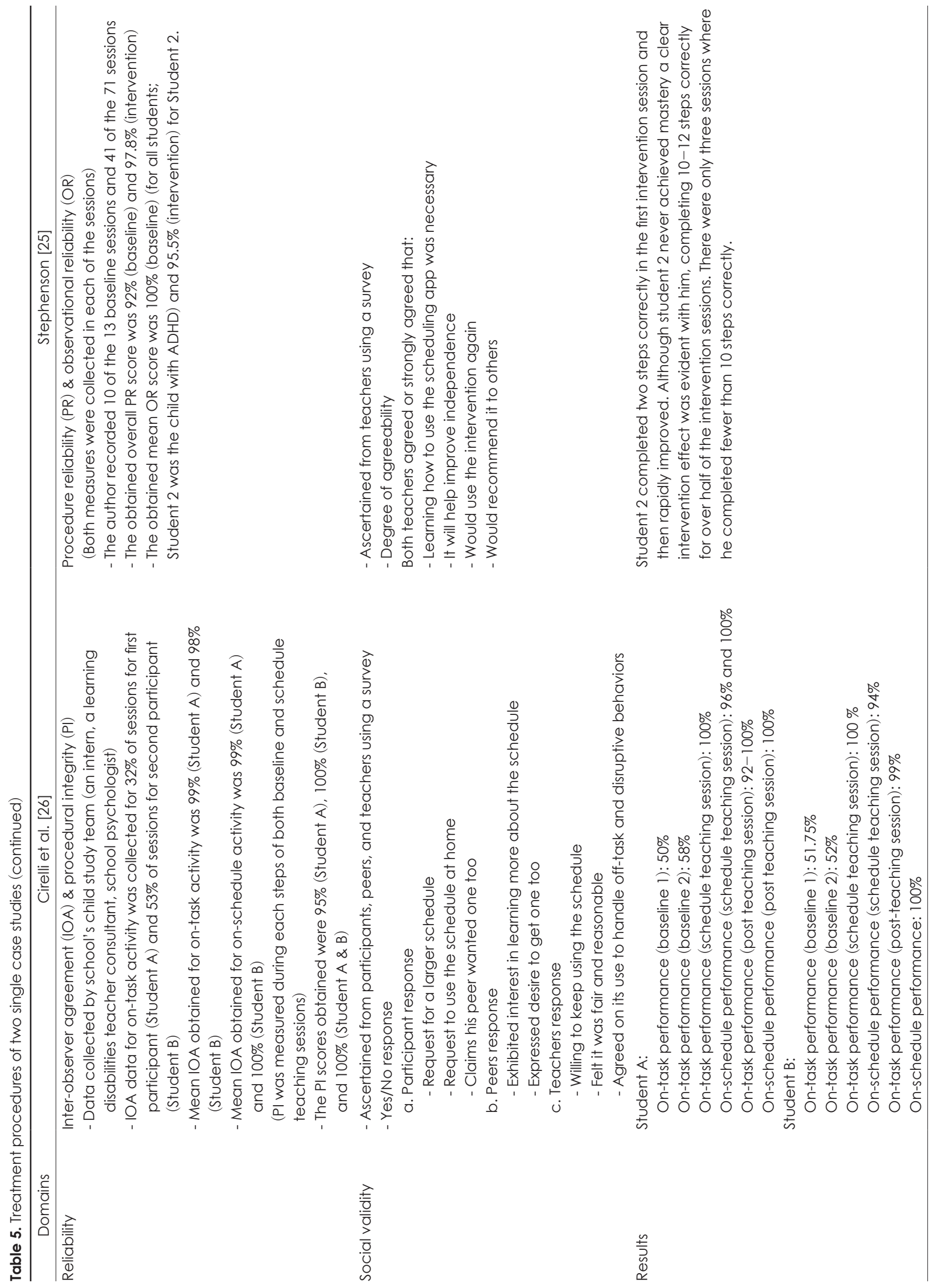




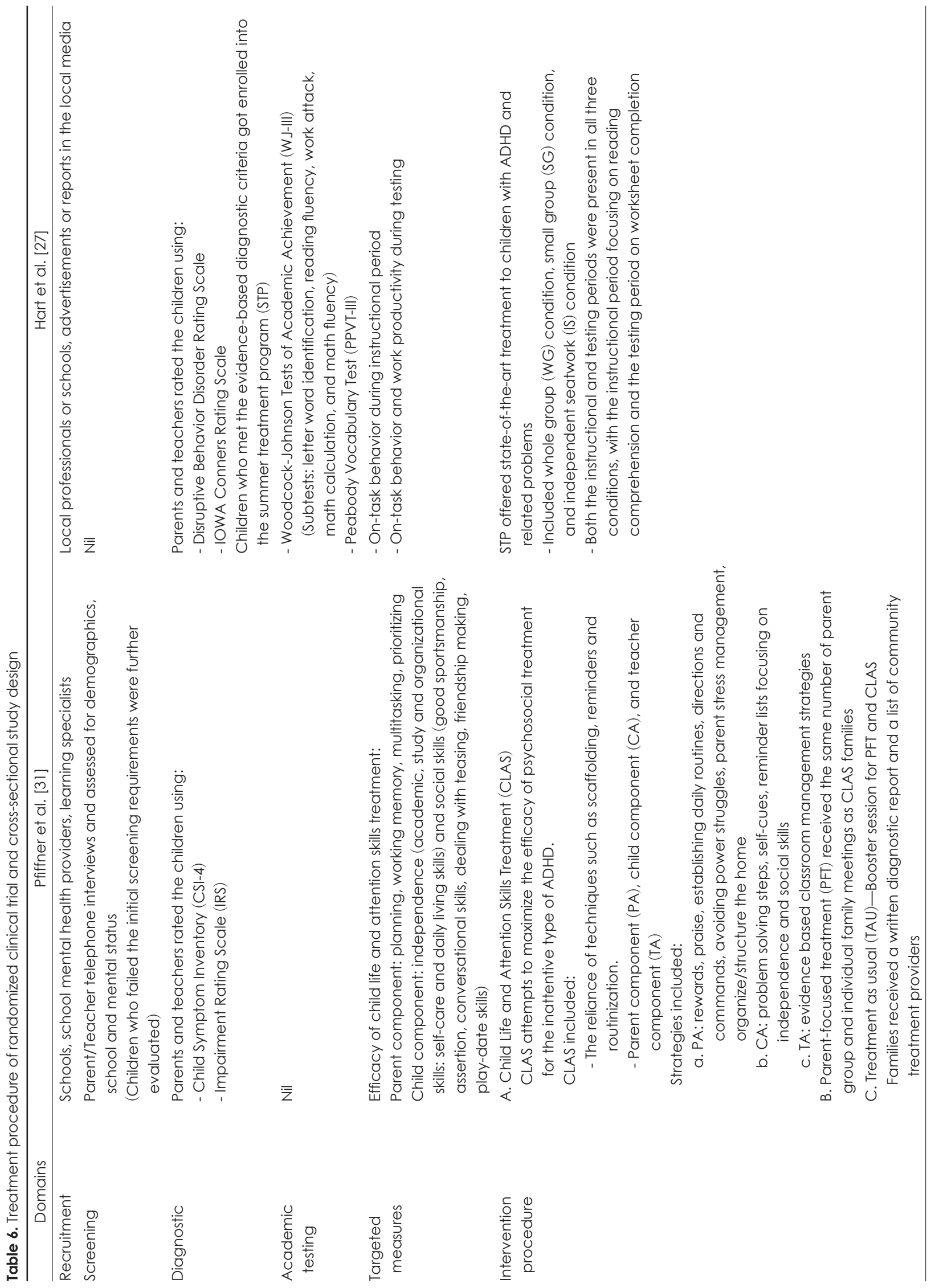




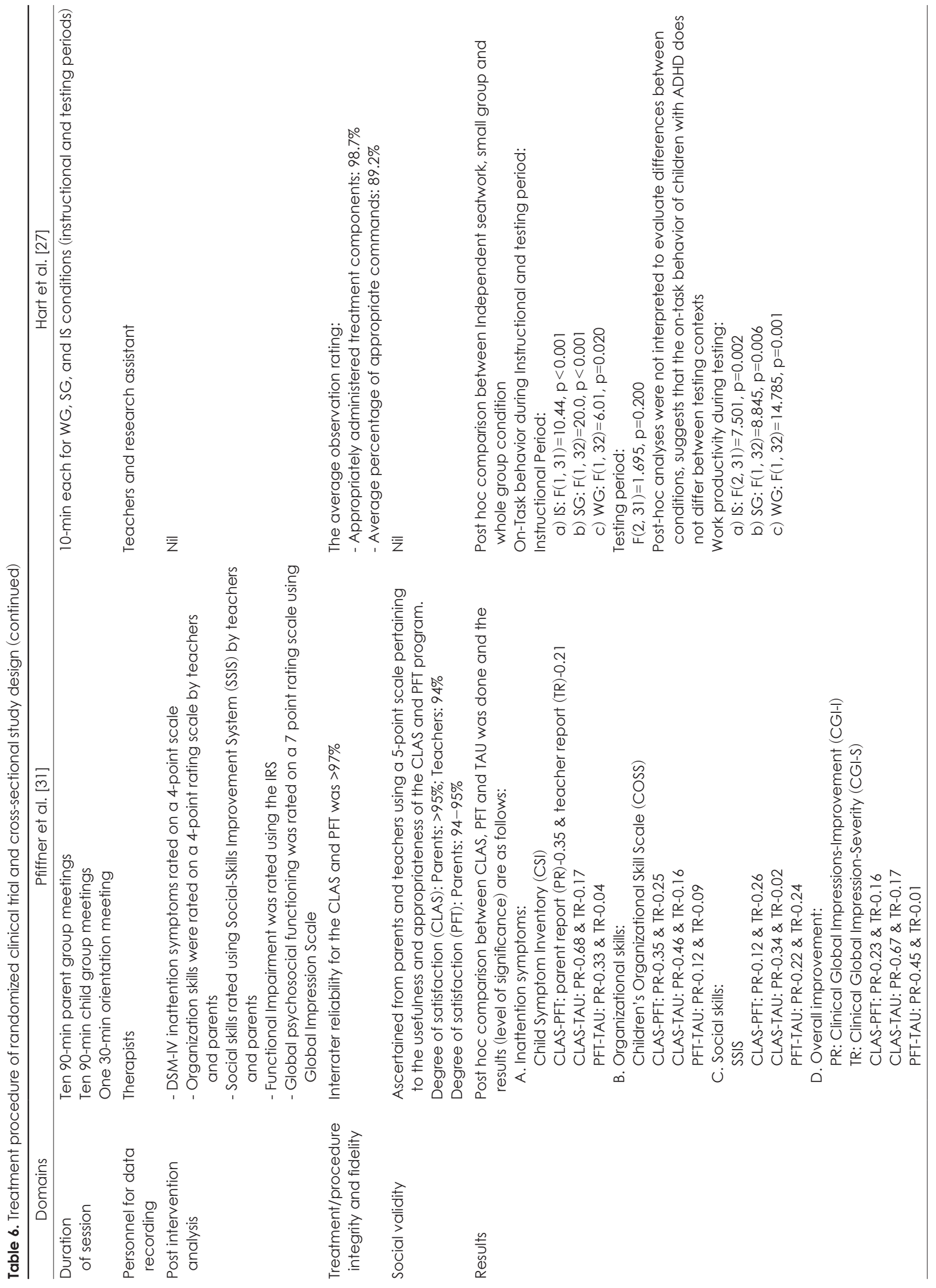


vention. In each of the studies, the duration of the VAS intervention program depended on the participants involved, task complexity, and materials used in the study. This indicates that VAS intervention programs that target training of stakeholders can be longer in duration and spread over a number of sessions, whereas programs directly targeting the ADHD students may be considered to be shorter in duration.

There were more than two team members conducting the therapy session, one of them could easily record the session, while the other concentrated on taking sessions, ensuring that there were no interruptions. It was important to evaluate the treatment program's efficacy or consistency to determine whether the intervention program was appropriate for treating the patients. In one of the single case studies [26] as well as in the RCT [31], the inter-observer reliability was rated. Simultaneously, procedural integrity, treatment fidelity, observational reliability, and procedural reliability were all critical in determining the effectiveness of the treatment program [40]. To prevent bias, all of these measures were included in the study [25-27]. The parents expressed high levels of satisfaction with the CLAS program as they learned more about ADHD and how to train their children with it [31]; however, there was no mention of social validity in another study [27]. Two of the studies $[25,26]$ assessed the acceptability of the treatment among teachers, patients, and peers, and found it to be satisfactory. The results of all four studies were measured differently depending on the type of intervention implemented. On-task and on-schedule performance were assessed on a percentage basis in one of the single case studies [26], while the progress in each step or session was examined in the other. The results of the RCT [31] were obtained by administering rating scales to the participants. The level of significance was measured in a cross-sectional study for all three conditions.

It was also crucial to conduct a post-intervention review to determine whether there were any deviations from the participant's baseline. The RCT [31] used various rating scales to perform a post-intervention review to see how far the participants had progressed or how different they were from their baseline phase. Various rating scales were used to assess the quality of the four studies [25-27,31]. The RCT [31] was of low quality, while the other three studies [25-27] were of high quality. Provided that all indicators had been similarly assessed, the efficiency of all four studies could have been ascertained uniformly.

\section{CONCLUSION}

The use of VAS in children with ADHD is a promising intervention that provides teachers and parents with adequate guidelines to execute the intervention program in different settings. Using VAS also seeks to improve the most impacted areas such as academic, social and independent classroom skills, on-task and on-schedule behavior, and literacy skills. The use of scheduled-based activities are found to have a significant effect on ADHD children, as per the reviewed studies [25-27,31]. The results of the current study will help provide practitioners with guidelines for preparing scientifically planned interventions for children with ADHD. However, the generalizability of the findings of the current systematic review towards a wider age group is questionable. Moreover, the four studies that were evaluated in this systematic review followed three different study designs, resulting in the efficacy of the intervention program being debatable. Future research could address these issues, as well as determine the efficacy of other intervention programs in children and individuals with ADHD.

\section{Availability of Data and Material}

Data sharing not applicable to this article as no datasets were generated or analyzed during the study.

\section{Conflicts of Interest}

The authors have no potential conflicts of interest to disclose.

\section{Author Contributions}

Conceptualization: Sudhin Karuppali. Data curation: Naveena Thomas. Formal analysis: all authors. Methodology: Naveena Thomas. Supervision: Sudhin Karuppali. Writing_original draft: Naveena Thomas. Writing_review \& editing: Sudhin Karuppali.

\section{ORCID iDs}

Naveena Thomas https://orcid.org/0000-0001-6107-0895

Sudhin Karuppali https://orcid.org/0000-0003-2955-8107

\section{Funding Statement}

None

\section{Acknowledgments}

The authors wish to thank Mr. Rahul K for acting as the third reviewer during the 'data extraction and management' and 'quality assessment' process.

\section{REFERENCES}

1) Ather M, Salmon G. Attention deficit hyperactivity disorder. Br J Hosp Med (Lond) 2010;71:641-644.

2) American Psychiatric Association. Diagnostic and statistical manual of mental disorders. 5th ed. Arlington, VA: American Psychiatric Association;2013.

3) Guevremont DC, Dumas MC. Peer relationship problems and disruptive behavior disorders. J Emot Behav Disord 1994;2:164-172.

4) Silva D, Colvin L, Glauert R, Stanley F, Srinivas Jois R, Bower C. Literacy and numeracy underachievement in boys and girls with ADHD. J Atten Disord 2020;24:1392-1402.

5) Hawkins E, Gathercole S, Astle D, Holmes J; The Calm Team. Language problems and ADHD symptoms: how specific are the links? Brain Sci 2016;6:50.

6) Arnett AB, Pennington BF, Willcutt E, Dmitrieva J, Byrne B, Sam- 
uelsson S, et al. A cross-lagged model of the development of ADHD inattention symptoms and rapid naming speed. J Abnorm Child Psychol 2012;40:1313-1326.

7) Korrel H, Mueller KL, Silk T, Anderson V, Sciberras E. Research review: language problems in children with attention-deficit hyperactivity disorder - a systematic meta-analytic review. J Child Psychol Psychiatry 2017;58:640-654.

8) Bruce B, Thernlund G, Nettelbladt U. ADHD and language impairment: a study of the parent questionnaire FTF (five to fifteen). Eur Child Adolesc Psychiatry 2006;15:52-60.

9) Catalá-López F, Hutton B, Núñez-Beltrán A, Mayhew AD, Page MJ, Ridao M, et al. The pharmacological and non-pharmacological treatment of attention deficit hyperactivity disorder in children and adolescents: protocol for a systematic review and network meta-analysis of randomized controlled trials. Syst Rev 2015;4:19.

10) Pelham WE Jr, Wheeler T, Chronis A. Empirically supported psychosocial treatments for attention deficit hyperactivity disorder. J Clin Child Psychol 1998;27:190-205.

11) Pfiffner LJ, Haack LM. Behavior management for school-aged children with ADHD. Child Adolesc Psychiatr Clin N Am 2014;23: 731-746.

12) Langberg JM, Epstein JN, Graham AJ. Organizational-skills interventions in the treatment of ADHD. Expert Rev Neurother 2008;8: $1549-1561$.

13) McCain AP, Kelley ML. Managing the classroom behavior of an ADHD preschooler. Child Fam Behav Ther 1993;15:33-44.

14) Knight V, Sartini E, Spriggs AD. Evaluating visual activity schedules as evidence-based practice for individuals with autism spectrum disorders. J Autism Dev Disord 2014;45:157-178.

15) Forness SR, Kavale KA, Bauman SSM. The psychiactric comorbidity hypothesis revisited. Learn Disabil Q 1998;21:203-206.

16) Dettmer S, Simpson RL, Myles BS, Ganz JB. The use of visual supports to facilitate transitions of students with autism. Focus Autism Other Dev Disabil 2000;15:163-169.

17) Schmit J, Alper S, Raschke D, Ryndak D. Effects of using a photographic cueing package during routine school transitions with a child who has autism. Ment Retard 2000;38:131-137.

18) Foorman BR, Torgesen J. Critical elements of classroom and smallgroup instruction promote reading success in all children. Learn Disabil Res Pract 2001;16:203-212.

19) Meindl JN, Delgado D, Casey LB. Increasing engagement in students with autism in inclusion classrooms. Child Youth Serv Rev 2020;111:104854

20) Aljubour A, AbdElBaki MA, El Meligy O, Al Jabri B, Sabbagh H. Effectiveness of dental visual aids in behavior management of children with autism spectrum disorder: a systematic review. Child Health Care 2020;50:83-107.

21) Balint-Langel K, Yuan C, Hua Y. Teaching the calendar application on a mobile device to young adults with intellectual and developmental disabilities. J Spec Educ Technol 2020 Jun 24 [Epub]. Availble from: https://journals.sagepub.com/doi/ abs/10.1177/0162643420933575.

22) Lequia J, Machalicek W, Rispoli MJ. Effects of activity schedules on challenging behavior exhibited in children with autism spectrum disorders: a systematic review. Res Autism Spectr Disord 2012; 6:480-492.

23) Bryan LC, Gast DL. Teaching on-task and on-schedule behaviors to high-functioning children with autism via picture activity schedules. J Autism Dev Disord 2000;30:553-567.
24) Larryant B. The impact of a visual activity schedule for teaching swimming to children with disabilities [dissertation]. Vancouver: British Columbia Univ.;2014.

25) Stephenson J. Teaching schedule use on an iPad to children with developmental disabilities. J Spec Educ Technol 2015;30:207-212.

26) Cirelli CA, Sidener TM, Reeve KF, Reeve SA. Using activity schedules to increase on-task behavior in children at risk for attentiondeficit/hyperactivity disorder. Educ Treat Child 2016;39:283-300.

27) Hart KC, Massetti GM, Fabiano GA, Pariseau ME, Pelham Jr WE. Impact of group size on classroom on-task behavior and work productivity in children with ADHD. J Emot Behav Disord 2011;19:5564.

28) Horner RH, Carr EG, Halle J, McGee G, Odom S, Wolery M. The use of single-subject research to identify evidence-based practice in special education. Except Child 2005;71:165-179.

29) Liberati A, Altman DG, Tetzlaff J, Mulrow C, Gøtzsche PC, Ioannidis JP, et al. The PRISMA statement for reporting systematic reviews and meta-analyses of studies that evaluate health care interventions: explanation and elaboration. J Clin Epidemiol 2009;62:e1e34.

30) Wells G, Shea B, O’Connell D, Robertson J, Peterson J, Welch V, et al. The Newcastle-Ottawa scale (NOS) for assessing the quality of nonrandomised studies in meta-analyses [Internet]. Ottawa: Ottawa Hospital Research Institute; 2011 [cited 2016 May 5]. Available from: http://www.evidencebasedpublichealth.de/download/ Newcastle_Ottowa_Scale_Pope_Bruce.pdf.

31) Pfiffner LJ, Hinshaw SP, Owens E, Zalecki C, Kaiser NM, Villodas M, et al. A two-site randomized clinical trial of integrated psychosocial treatment for ADHD-inattentive type. J Consult Clin Psychol 2014;82:1115-1127.

32) Yang ZR, Sun F, Zhan SY. [Risk on bias assessment: (2) Revised Cochrane risk of bias tool for individually randomized, parallel group trials (RoB2.0)]. Zhonghua Liu Xing Bing Xue Za Zhi 2017;38:12851291.

33) Conners CK, Sitarenios G, Parker JD, Epstein JN. Revision and restandardization of the Conners Teacher Rating Scale (CTRS-R): factor structure, reliability, and criterion validity. J Abnorm Child Psychol 1998;26:279-291.

34) DuPaul GJ, Power TJ, Anastopoulos AD, Reid R, McGoey KE, Ikeda MJ. Teacher ratings of attention deficit hyperactivity disorder symptoms: factor structure and normative data. Psychol Assess 1997;9:436-444.

35) Goyette CH, Conners CK, Ulrich RF. Normative data on revised conners parent and teacher rating scales. J Abnorm Child Psychol 1978;6:221-236.

36) Nathan RA. How important is patient recruitment in performing clinical trials? J Asthma 1999;36:213-216.

37) Felver JC, Celis-de Hoyos CE, Tezanos K, Singh NN. A systematic review of mindfulness-based interventions for youth in school settings. Mindfulness 2016;7:34-45.

38) Lin-Siegler X, Dweck CS, Cohen GL. Instructional interventions that motivate classroom learning. J Educ Psychol 2016;108:295-299.

39) Van Geel M, Keuning T, Visscher AJ, Fox JP. Assessing the effects of a school-wide data-based decision-making intervention on student achievement growth in primary schools. Am Educ Res J 2016; 53:360-394.

40) Crane RS, Hecht FM. Intervention integrity in mindfulness-based research. Mindfulness (N Y) 2018;9:1370-1380. 